\title{
On the Validity of the "Thin" and "Thick" Double-Layer Assumptions When Calculating Streaming Currents in Porous Media
}

\author{
Matthew D. Jackson and Eli Leinov \\ Department of Earth Science and Engineering, Imperial College London, London SW7 2AZ, UK \\ Correspondence should be addressed to Matthew D. Jackson, m.d.jackson@imperial.ac.uk
}

Received 10 August 2011; Revised 1 December 2011; Accepted 19 January 2012

Academic Editor: Laurence Jouniaux

Copyright ( 2012 M. D. Jackson and E. Leinov. This is an open access article distributed under the Creative Commons Attribution License, which permits unrestricted use, distribution, and reproduction in any medium, provided the original work is properly cited.

We find that the thin double layer assumption, in which the thickness of the electrical diffuse layer is assumed small compared to the radius of curvature of a pore or throat, is valid in a capillary tubes model so long as the capillary radius is $>200$ times the double layer thickness, while the thick double layer assumption, in which the diffuse layer is assumed to extend across the entire pore or throat, is valid so long as the capillary radius is $>6$ times smaller than the double layer thickness. At low surface charge density $\left(<10 \mathrm{mC} \cdot \mathrm{m}^{-2}\right)$ or high electrolyte concentration $(>0.5 \mathrm{M})$ the validity criteria are less stringent. Our results suggest that the thin double layer assumption is valid in sandstones at low specific surface charge $\left(<10 \mathrm{mC} \cdot \mathrm{m}^{-2}\right)$, but may not be valid in sandstones of moderate- to small pore-throat size at higher surface charge if the brine concentration is low $(<0.001 \mathrm{M})$. The thick double layer assumption is likely to be valid in mudstones at low brine concentration $(<0.1 \mathrm{M})$ and surface charge $\left(<10 \mathrm{mC} \cdot \mathrm{m}^{-2}\right)$, but at higher surface charge, it is likely to be valid only at low brine concentration $(<0.003 \mathrm{M})$. Consequently, neither assumption may be valid in mudstones saturated with natural brines.

\section{Introduction}

Streaming potentials in porous materials arise from the electrical double layer which forms at solid-fluid interfaces (e.g., [1]). The solid surfaces typically become electrically charged, in which case an excess of countercharge accumulates in the adjacent fluid, in an arrangement called the electrical double layer. The double layer comprises an inner compact (Stern) layer and an outer diffuse (Gouy-Chapman) layer. Most of the countercharge typically resides within the Stern layer; however, if the fluid is induced to flow by an external pressure gradient, then some of the excess charge within the diffuse layer is transported with the flow, giving rise to a streaming current. Divergence of the streaming current density establishes an electrical potential, termed the streaming potential (e.g., [2-4]).

Within the diffuse layer, the Poisson-Boltzmann equation is typically used to describe the variation in electrical potential with distance from the solid surface; in cylindrical coordinates and assuming a symmetric, monovalent electrolyte, the Poisson-Boltzmann equation is given in dimensionless form by [5]

$$
\frac{1}{R} \frac{\partial}{\partial R}\left(R \frac{\partial \psi}{\partial R}\right)=\left(\frac{r}{\lambda}\right)^{2} \sinh (\psi),
$$

where the dimensionless electrical potential is $\psi \equiv V /(k T / e)$, and dimensionless radial position is $R \equiv y / r$ (Figure 1 ) (see Table 1 for the nomenclature). The electrical potential is denoted $V, T$ is temperature, $k$ is Boltzmann's constant, $e$ is the electron charge, $\lambda$ is a characteristic lengthscale termed the Debye length, and $r$ is the radius of a cylindrical pore (capillary). The Debye length is a measure of the diffuse layer thickness; its value depends upon the concentration of the salt species and, assuming (1) is valid, is given by

$$
\lambda=\left(\frac{2000 N_{\mathrm{A}} C_{f} e^{2}}{\varepsilon k T}\right)^{-1 / 2},
$$

where $N_{\mathrm{A}}$ is Avogadro's number, $C_{f}$ is molar concentration, and $T$ is temperature (e.g., [2]). The derivation of (1) 


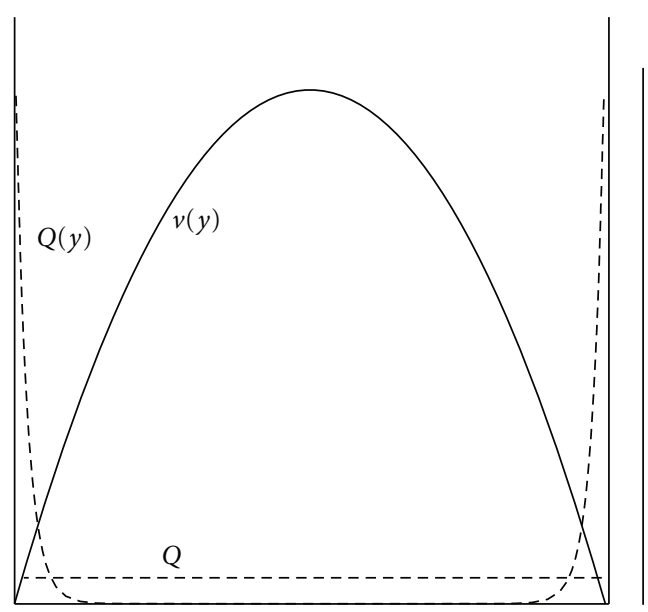

(a)

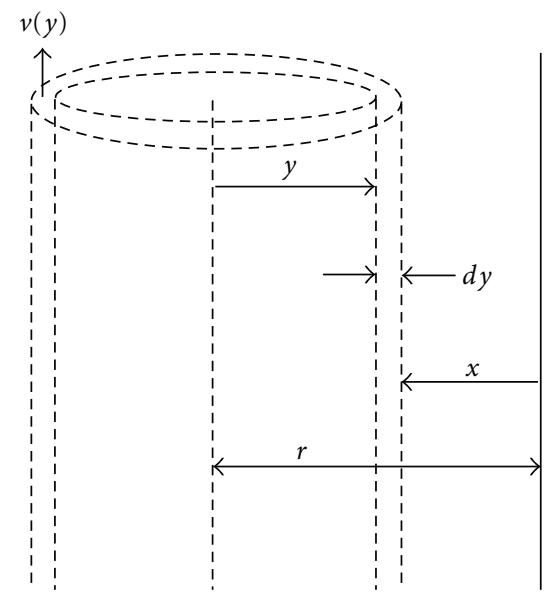

(b)

FIGURE 1: (a) Flow velocity (solid line) and excess countecharge within a capillary, invoking the "thin" (short dashes) and "thick" (long dashes) double-layer assumption and assuming a constant excess surface charge density. The width of the "thin" double-layer has been greatly exaggerated. The excess charge on the capillary surface, and hence the total excess countercharge within the capillary, are the same for both the "thin" and "thick" double layer assumptions; the only difference between the models is the distribution of the countercharge within the capillary. (b) Calculation of the streaming current. Assuming Poiseuille flow, each cylinder of fluid of thickness $d y$ travels at velocity $v(y)$ and transports an excess charge density $Q(y)$. The distribution of excess charge within the capillary impacts on the streaming current because excess charge at the centre of the capillary is transported more rapidly by the flow than excess charge at the margin of the capillary. Modified from Jackson [6].

assumes that the ions are point charges, the fluid is continuous and characterized by a constant permittivity which is not affected by the overall electrical field strength, and that the only work done in bringing an ion to a location in the electrical field is related to the field and not to displacement of the fluid or interactions with other ions (e.g., [2]). The Debye length ranges from c. $1 \mathrm{~nm}$ in a monovalent electrolyte at $0.1 \mathrm{M}$ concentration, to c. $10 \mathrm{~nm}$ at a concentration of $0.001 \mathrm{M}$, and is independent of the radius of the pore or capillary; if the Debye length exceeds the pore radius, there is double-layer overlap (e.g., [2, pages 363-369]).

In many porous materials of interest to earth and material scientists, it is reasonable to assume that the thickness of the diffuse layer $(\lambda)$ is small compared to the pore radius $(r)$. This is the so-called "thin double layer assumption" which is valid when $r \gg \lambda$. Under this limiting condition, curvature of the pore surface can be neglected, and the equations describing fluid flow can be linearized in the region of the pore surface. The thin double-layer assumption has been invoked by numerous authors to model streaming potentials in porous media (e.g., $[3,4,11-14])$ and is also invoked in the derivation of the widely applied Helmholtz-Smoluchowski equation (see [2], and references therein):

$$
C=\frac{\varepsilon \zeta}{\mu \sigma_{f}},
$$

where $C$ is the streaming potential coupling coefficient, $\zeta$ is the zeta potential, which is the electrical potential defined at the inner boundary of the mobile part of the diffuse layer, $\varepsilon$ is the fluid permittivity, $\mu$ is the fluid viscosity, and $\sigma_{f}$ is the fluid electrical conductivity.

The Helmholtz-Smoluchowski equation has been used to determine the zeta potential from streaming potential measurements in numerous studies (see [15], for a review]) with appropriate corrections to account for surface electrical conductivity (e.g., $[13,16,17])$. However, a number of recent papers have proposed a different approach to model streaming potential, in which the thickness of the double layer is assumed to be large compared to the radius of the pores (i.e., the Debye length is much greater than the pore radius) $[18,19]$. This is the so-called "thick double layer assumption" in which the excess charge density within the pore $(Q)$ is assumed to be constant and independent of distance from the pore surface. The streaming potential coupling coefficient is then given by

$$
C=\frac{Q \kappa}{\mu \sigma_{f}}
$$

where $\kappa$ is the permeability of the porous material (e.g., [18]).

The advantage of both the "thin" and "thick" double layer assumptions is that calculation of the streaming current is greatly simplified because there is no need to explicitly solve the Poisson-Boltzmann equation (1). Obtaining such solutions is challenging, especially when the pore space has a complex topology; analytical solutions are available only for some restricted cases (e.g., [2], and references therein, $[20,21])$. In the thin double-layer assumption, the PoissonBoltzmann equation is linearized in the region close to the pore surface; in the thick double-layer assumption, the excess charge is assumed to be uniformly distributed across each pore or throat.

It is well known that the thin double-layer assumption is valid if the thickness of the double-layer is "much less" than the radius of the pore or throat $(r \gg \lambda)$, while the thick double layer assumption is valid if the thickness of 
TABLE 1: Nomenclature.

\begin{tabular}{|c|c|c|}
\hline Symbol & Description & Units \\
\hline$C_{f}$ & Fluid concentration & M \\
\hline$e$ & Charge on an electron & $\mathrm{C}$ \\
\hline$\varepsilon$ & Fluid permittivity & $\mathrm{F} \mathrm{m}^{-1}$ \\
\hline$I$ & Electrical (streaming) current & A \\
\hline$I_{d}$ & Dimensionless streaming current & - \\
\hline$k$ & Boltzmann's constant & $\mathrm{m}^{2} \mathrm{~kg} \mathrm{~s}^{-2} \mathrm{~K}^{-1}$ \\
\hline$\kappa$ & Permeability & $\mathrm{m}^{2}$ \\
\hline$L$ & Capillary length & $\mathrm{m}$ \\
\hline$\lambda$ & Debye length & $\mathrm{m}$ \\
\hline$\mu$ & Fluid viscosity & Pas \\
\hline$N_{\mathrm{A}}$ & Avogradro's number & $\mathrm{Mol}^{-1}$ \\
\hline$P$ & Fluid pressure & $\mathrm{Pa}$ \\
\hline$q$ & Volumetric flow rate & $\mathrm{m}^{3} \mathrm{~s}^{-1}$ \\
\hline$Q$ & Excess charge density & $\mathrm{C} \mathrm{m}^{-3}$ \\
\hline$Q_{s}$ & Surface charge density & $\mathrm{Cm}^{-2}$ \\
\hline$Q_{d}$ & $\begin{array}{l}\text { Dimensionless surface charge } \\
\text { density }\end{array}$ & - \\
\hline$r$ & Capillary radius & $\mathrm{m}$ \\
\hline$r_{d}$ & Dimensionless capillary radius & - \\
\hline$R$ & Dimensionless radial position & - \\
\hline$\sigma_{f}$ & Fluid conductivity & $\mathrm{Sm}^{-1}$ \\
\hline$T$ & Temperature & K \\
\hline$V$ & Electrical potential & $\mathrm{V}$ \\
\hline$\psi$ & $\begin{array}{l}\text { Dimensionless electrical } \\
\text { potential }\end{array}$ & - \\
\hline$y$ & Radial position & $\mathrm{m}$ \\
\hline$\zeta$ & Zeta potential & $\mathrm{V}$ \\
\hline
\end{tabular}

the double layer is "much greater" than the radius of the pore or throat $(r \ll \lambda)$. However, perhaps surprisingly, the conditions for which these assumptions are valid have not yet been determined quantitatively. Yet the two models predict different streaming potential behaviour for a given specific surface charge $\left(Q_{s}\right)$ because excess charge at the centre of a pore or throat is transported more rapidly by the flow than excess charge at the margin of a pore or throat $[6$, see Figure 1]. Moreover, porous materials encountered in both earth and materials science cover a broad range of pore size $(r)$ and are saturated with fluids of varying salt concentration (salinity) which, assuming the electrical potential in the diffuse layer is described by (1), controls the thickness of the diffuse layer $(\lambda)$ through (2). Consequently, the assumption of a thin or thick double layer may often be invalid.

Westermann-Clark and Christoforou [5] compared the exclusion-diffusion potential predicted across a single capillary, using a space-charge model that includes a numerical solution of the Poisson-Boltzmann equation, with that obtained using the Meyers-Sievers model, in which the excess charge density is assumed to be independent of distance from the pore surface. They found that the Meyers-Sievers model was a good approximation of the space-charge model when (i) the pore surface charge is small, (ii) when $r / \lambda \rightarrow 0$, and (iii) when $r / \lambda \rightarrow \infty$. The Meyers-Sievers model is equivalent to the thick double-layer assumption when $r / \lambda \rightarrow$ 0 . However, it is not equivalent to the thin double-layer assumption when $r / \lambda \rightarrow \infty$. In the Meyers-Sievers model, the diffuse layer thickness is zero in this limit, in which case there is no streaming current and no streaming potential. Consequently, the results of Westermann-Clark and Christoforou [5] cannot be used to determine the validity of the thin and thick double-layer assumptions when calculating streaming current. The thin double-layer assumption invokes a small but nonzero diffuse layer thickness, which gives rise to non-zero streaming potentials. Experimental evidence for a non-zero diffuse layer thickness, even at high ionic strength when (2) predicts that $\lambda$ becomes infinitesimally small, has been provided by Dukhin et al. [22] and Vinogradov et al. [23].

The aim of this paper is to determine the conditions for which it is valid to invoke the thin and thick double-layer assumptions when calculating the streaming current in a simple bundle of capillary tubes model. Although it is a poor representation of the pore space of most geologic porous media, the advantage of a capillary tubes model is that the capillary scale distribution and transport of excess charge associated with the electrical double-layer is easy to describe; capillary models have been used to calculate the streaming potential in numerous previous studies (see [2], and references therein; [6, 24-26]). Our approach combines those of Jackson [6] and Westermann-Clarke and Christoforou [5]. We investigate the validity of streaming current calculations, rather than streaming potential calculations, because the former is an essential step in calculating the latter, but the latter also requires a model for the electrical conductivity, which is consistent with the pore level distribution of excess charge. The development of such a model is left for future study.

\section{Model Formulation}

The capillary tubes model is simplified from that described in detail by Jackson $[6,25]$, so only a brief overview is provided here. Each capillary has the same length $L$, radius $r$, and orientation; there are no intersections between capillaries, so the macroscopic mass and charge transport are in one direction only. The charge per unit surface area (the specific surface charge) $Q_{s}$ is the same in all capillaries, which is reasonable so long as the chemical composition of the fluid and the capillary surfaces do not vary between capillaries. The specific surface charge $\left(Q_{s}\right)$ is evenly distributed along the capillary surfaces and incorporates the contribution of adsorbed charge within the Stern layer; this is equivalent to defining the capillary surface to be the plane separating the Stern and diffuse layers. To maintain a consistent model, we assume that the radius of each capillary $(r)$ is defined between its centre and this plane, which is equivalent to defining the capillary surface to be the shear plane. Since the capillaries are identical, the model is analyzed as if it were a single capillary. A similar approach was followed by Westermann-Clark and Christoforou [5]. 
The streaming current is calculated assuming laminar flow, in which each concentric cylinder of fluid moving with velocity $v(y)$ along the capillary, transports an excess charge density $Q(y)$ (Figure 1). The fluid velocity is given by Poiseulle's Law:

$$
v(y)=\frac{1}{4 \mu}\left(r^{2}-y^{2}\right) \frac{\Delta P}{L},
$$

where $\Delta P$ is the pressure drop along the capillary, and $L$ is the length of the capillary. The streaming current is given by $[2$, page 65]:

$$
I=\int_{0}^{r} 2 \pi y Q(y) v(y) d y,
$$

where we neglect the impact on the streaming current of the electrical potential difference along the capillary [27]. The description of the excess charge density $Q(y)$ depends upon whether we invoke the thin or thick double-layer assumptions, or explicitly model $Q(y)$ using the Poisson-Boltzmann equation.

We begin by invoking the thin double-layer assumption and, for simplicity, the Debye-Hückel approximation in which the electrical potential within the double layer is assumed to be small $\left(<25.7 \mathrm{mV}\right.$ at $25^{\circ} \mathrm{C}$; Hunter [2]). The excess charge density within the diffuse layer can then be described as a function of distance from the capillary surface using

$$
Q(x)=Q(x=0) \exp \left(-\frac{x}{\lambda}\right),
$$

where $Q(x=0)$ is the excess charge density in the fluid at the capillary surface (which is not equal in magnitude to the surface charge density) and $x=(r-y)$ [2]. The thickness of the diffuse layer is much less than the capillary radius $(\lambda \ll r)$, so the velocity profile in the diffuse layer close to the capillary surface can be assumed linear by taking

$$
\begin{gathered}
\left(r^{2}-y^{2}\right) \approx 2 r(r-y), \\
r^{2}\left(\frac{x-x^{2}}{r}\right) \approx r^{2} x,
\end{gathered}
$$

(see Hunter [2, page 66]). The streaming current can then be written as ([2, equation 3.2.2])

$$
I=\frac{Q(x=0) \pi r^{2} \Delta P}{\mu L} \int_{0}^{r} x \exp \left(-\frac{x}{\lambda}\right) d x .
$$

Integrating by parts and recognizing that $Q(x)$ is zero at $x=r$ (indeed, long before $x=r$ ) the streaming current through a single capillary becomes

$$
I=-\frac{Q(x=0) \pi \lambda^{2} r^{2} \Delta P}{\mu L} .
$$

We can express (10) in terms of the surface charge density by recognizing that the total surface charge on the capillary must be balanced by the excess countercharge within the fluid occupying the capillary:

$$
2 \pi r Q_{s} L=-L \int_{0}^{r} 2 \pi y Q(y) d y .
$$

Given that $\lambda \ll r$, this yields

$$
\left.I\right|_{\lambda \ll r}=-\frac{Q_{s} \pi \lambda r^{2} \Delta P}{\mu L} .
$$

Equation (12) describes the streaming current through a capillary tube of radius $r$, assuming a thin electrical double layer for which the Debye-Hückel approximation is valid, associated with specific surface charge $Q_{s}$. It is easy to show that (12) can also be expressed in terms of the zeta potential at the shear plane (see, e.g., (3.2.3) in Hunter [2]), rather than the specific surface charge.

We now invoke the thick double-layer assumption. The excess charge density in a given phase is constant across the capillary, in which case the streaming current can be written as

$$
I=-\frac{Q \pi r^{4} \Delta P}{8 \mu L} .
$$

As before, we can express (13) in terms of the surface charge density using (11) to give

$$
\left.I\right|_{\lambda \gg r}=-\frac{Q_{s} \pi r^{3} \Delta P}{4 \mu L} .
$$

Equation (14) describes the streaming current through a capillary tube of radius $r$, assuming a thick electrical double layer associated with specific surface charge $Q_{s}$.

Finally, we consider explicit solutions to the PoissonBoltzmann equation to determine $Q(y)$. In cylindrical coordinates, suitable for a capillary tube model, the PoissonBoltzmann equation is given by (1). There is no variation in electrical potential along the axis of the capillary because there is no concentration difference. The excess charge at a dimensionless radial position $R \equiv y / r$ is related to the dimensionless potential $\psi$ by

$$
Q(R)=2000 e C_{f} N_{\mathrm{A}} \sinh (\psi(R)) .
$$

Substituting (15) into (6) and simplifying yields the following expression for the streaming current:

$$
I=\frac{1000 e C_{f} N_{\mathrm{A}} \pi r^{4} \Delta P}{\mu L} \int_{0}^{1} R \cdot \sinh (\psi(R)) \cdot\left(1-R^{2}\right) d R,
$$

with the dimensionless electrical potential $(\psi)$ given by (1). There are no exact analytical solutions to (1), so we use a modified implicit Runge-Kutta scheme with residual control (see [28]) to obtain numerical solutions subject to the following boundary conditions at the shear plane $(R=1)$ and at the centre of the capillary $(R=0)[5]$ :

$$
\begin{gathered}
\left.\frac{\partial \psi}{\partial R}\right|_{R=0}=0, \\
\left.\frac{\partial \psi}{\partial R}\right|_{R=1}=Q_{d},
\end{gathered}
$$


where $Q_{d}$ is the dimensionless surface charge density, defined as

$$
Q_{d}=-\frac{e r Q_{s}}{\varepsilon k T}
$$

Having determined $\psi(R)$, we integrate (16) numerically over $R$ to determine $I_{s}$. Note that the boundary condition (17a) allows us to account for double-layer overlap in our solutions of the Poisson-Boltzmann equation (1).

The behaviour of the dimensionless electrical potential and hence the excess charge, as a function of the radial position, is governed by the dimensionless pore wall surface charge density $\left(Q_{d}\right)$ and the dimensionless capillary radius $\left(r_{d}\right)$. The latter is defined as the ratio of the capillary radius to the Debye length:

$$
r_{d}=\frac{r}{\lambda}
$$

As $r_{d} \rightarrow 0$, the double-layer thickness becomes very large compared to the capillary radius $(r \ll \lambda$; the limit of a thick double layer), while as $r_{d} \rightarrow \infty$, the double layer thickness becomes very small compared to the capillary radius $(r \gg \lambda$; the limit of a thin double layer). We quantify the validity of the thin and thick double layer assumptions by plotting dimensionless streaming current $\left(I_{d}\right)$ as a function of dimensionless capillary radius $\left(r_{d}\right)$. The dimensionless streaming current is given by

$$
I_{d}=\frac{I\left(r_{d}\right)-\left.I\right|_{\lambda \gg r}}{\left.I\right|_{\lambda \ll r}-\left.I\right|_{\lambda \gg r}}
$$

where $I\left(r_{d}\right)$ is given by the numerical solution of (16), $\left.I\right|_{\lambda \ll r}$ is the streaming current in the limit of a thin double layer (12), and $\left.I\right|_{\lambda \gg r}$ is the streaming current in the limit of a thick double layer (14). If $I_{d}=0$, the streaming current calculated by solving the Poisson-Boltzmann equation to determine $Q(R)$ is identical to that obtained assuming a thick double layer, while if $I_{d}=1$, the streaming current calculated by solving the Poisson-Boltzmann equation is identical to that obtained assuming a thin double layer.

We select values of surface charge $\left(Q_{s}\right)$ and concentration $\left(C_{f}\right)$ and hold these constant whilst varying the value of $r$ to investigate how the dimensionless streaming current $\left(I_{d}\right)$ varies as a function of $r_{d}$. We assume the doublelayer thickness $(\lambda)$ is related to concentration via (2). We investigate concentration $\left(C_{f}\right)$ over the range $10^{-3}-2 \mathrm{M}$ $\left(\mathrm{mol} \cdot \mathrm{L}^{-1}\right)$ and specific surface electrical charge $\left(Q_{s}\right)$ over the range $1-100 \mathrm{mC} \cdot \mathrm{m}^{-2}$ to capture the range typically observed in natural systems and laboratory membranes. We discuss the validity of the Poisson-Boltzmann equation (1) over the concentration range investigated, and its impact on our results, later in the paper. Surface charge and electrolyte concentration are varied independently, although the former may depend upon the latter (e.g., $[3,21,29]$ ). The $\mathrm{pH}$ is assumed to remain fixed at 7 . Our definition of surface charge includes the contribution of adsorbed charge within the Stern layer, and the range we investigate is based on (i) published values for quartz and clay minerals in contact with $\mathrm{NaCl}$ brine (e.g., $[18,21,29]$ ) and (ii) published zeta potential data (see Vinogradov et al. [23] for a compilation of values on quartz and glass in $\mathrm{NaCl}$ electrolyte, and Kosmulski and Dahlsten [30] for values of clay minerals in $\mathrm{NaCl}$ electrolyte), with surface charge related to zeta potential using the Gouy-Chapman model [2]:

$$
Q_{s}=2 \sqrt{2000 k T C_{f} N_{\mathrm{A}} \varepsilon} \sinh \left(\frac{e \zeta}{2 k T}\right) .
$$

Measured values of zeta potential on quartz and clay minerals vary in magnitude from c. $100 \mathrm{mV}$ (at low concentration) to c. $2 \mathrm{mV}$ (at high concentration) over the concentration range investigated here; corresponding values of surface charge density, calculated using (21), lie well within the range we have chosen to investigate and are consistent with published values. Note that the results of our analysis depend only upon the magnitude of the surface charge, not its polarity. The dimensionless surface charge density $\left(Q_{d}\right)$ depends on the fluid permittivity, which varies with concentration (18). We capture this using

$$
\varepsilon=8.85 \times 10^{-12}\left(80-13 C_{f}+1.065 C_{f}^{2}-0.03006 C_{f}^{3}\right),
$$

where concentration is in $M$, and permittivity is in $\mathrm{F} \cdot \mathrm{m}^{-1}$ [31].

Note that our approach, in which we hold $Q_{s}$ and $C_{f}$ (and therefore $\lambda$ ) constant and vary $r_{d}$, differs from that of Westermann-Clark and Christoforou [5]. They held $Q_{d}$ constant and varied $r_{d}$ without acknowledging that this requires $Q_{s}$ to vary as $r_{d}$ varies, regardless of whether $r_{d}$ is varied by changing the capillary radius $(r)$ or diffuse layer thickness $(\lambda)$. Variations in $\lambda$ are associated with changes in concentration that impact on permittivity $(\varepsilon)$ and hence $Q_{d}$ (18); variations in $r$ require variations in $Q_{s}$ to maintain constant $Q_{d}(18)$. Assuming constant $Q_{s}$ whilst varying $r$ is more physically plausible than assuming constant $Q_{d}$.

\section{Results}

The variation in excess charge with distance from the capillary wall is shown in Figure 2, for a capillary radius of $r=4 \mathrm{~nm}$, two values of $Q_{s}$ reasonable for geologic porous media (Table 2), and two values of $C_{f}$ chosen to yield $\lambda \ll r$ at high concentration and $\lambda \gg r$ at low concentration. Also shown is the variation in excess charge with distance, calculated assuming a thin and thick double layers. The latter is constant and given by

$$
Q(x)=\frac{Q_{s}}{r}
$$

The former is obtained using two models: the Debye-Hückel model which assumes that $Q_{s}$ is $\operatorname{small}\left(<13.9 \mathrm{mC} \cdot \mathrm{m}^{-2}\right.$ at 


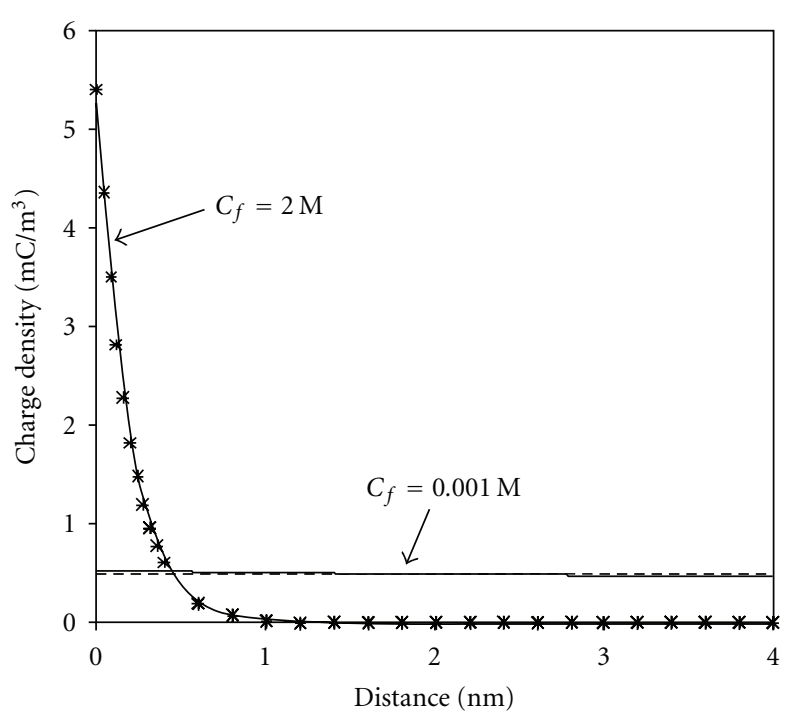

(a)

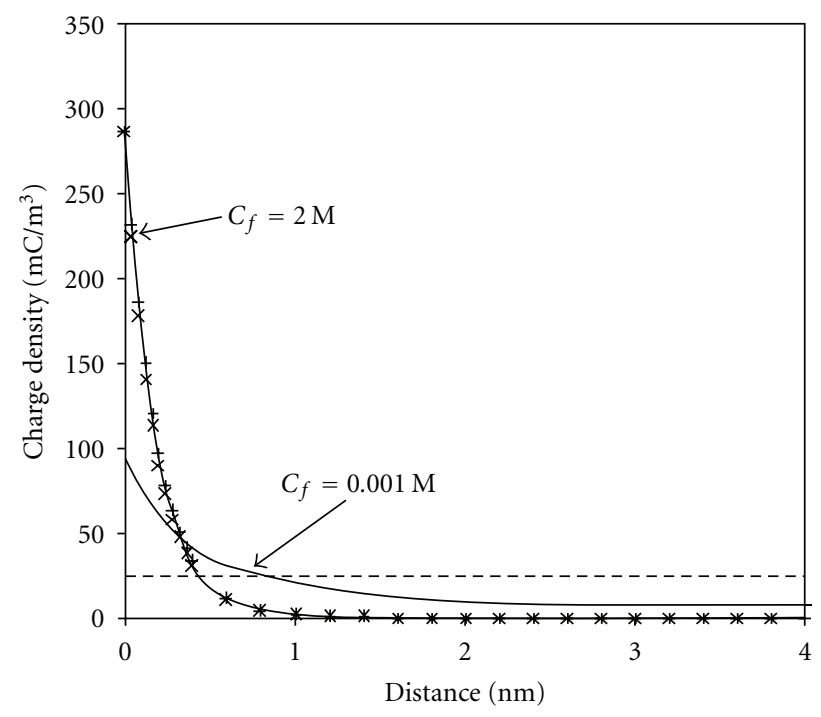

(b)

Figure 2: Excess charge density $(Q)$ as a function of distance from the capillary centre $(x)$ in a circular capillary of radius $4 \mathrm{~nm}$, for a surface charge of (a) $1 \mathrm{mC} \cdot \mathrm{m}^{-2}$ and (b) $50 \mathrm{mC} \cdot \mathrm{m}^{-2}$. Each plot shows results for electrolyte salinities of $0.001 \mathrm{M}$ and $2 \mathrm{M}$, corresponding to dimensionless capillary radii of 0.41 (i.e., towards the limit for which the thick double layer assumption is valid) and 21.6 (i.e., towards the limit for which the thin double-layer assumption is valid). Solid lines show $Q$ obtained from a numerical solution of the Poisson-Boltzmann equation (1) in conjunction with (16); crosses show $Q$ obtained from an analytic solution to the Poisson-Boltzmann equation assuming a thin double-layer (diagonal crosses show the Gouy-Chapman model given by (24); vertical crosses show the Debye-Hückel model given by (7)); dashed lines show constant $Q$ assuming a thick double layer.

$25^{\circ} \mathrm{C}$, corresponding to a zeta potential of $25.7 \mathrm{mV}$ ) and is given by (7), and the Gouy-Chapman model [2]:

$$
Q(x)=2000 e C_{f} N_{\mathrm{A}} \sinh \left[-2 \ln \left(\frac{1+\exp (-x / \lambda) \tanh \left((1 / 2) \sinh ^{-1}\left(Q_{s} / 2 \sqrt{2000 k T C_{f} N_{\mathrm{A}} \varepsilon}\right)\right)}{1-\exp (-x / \lambda) \tanh \left((1 / 2) \sinh ^{-1}\left(Q_{s} / 2 \sqrt{2000 k T C_{f} N_{\mathrm{A}} \varepsilon}\right)\right)}\right)\right]
$$

The results shown in Figure 2 confirm that the excess charge density calculated numerically tends towards the constant value given by (23) when $\lambda \gg r$, while the excess charge calculated numerically agrees with the values given by (7) and (24) when $\lambda \ll r$. This suggests that our numerical solutions to the Poisson-Boltzmann equation yield accurate results. Also note that the Debye-Hückel model provides a reasonably close match to the GouyChapman model up to the largest value of surface charge investigated $\left(100 \mathrm{mC} \cdot \mathrm{m}^{-2}\right)$, even though it is strictly valid only for $Q_{s}<13.9 \mathrm{mC} \cdot \mathrm{m}^{-2}$. In this study, the DebyeHückel model provides an appropriate limiting case for the thin double-layer approximation when calculating the dimensionless streaming current.

Figure 3 shows the variation of dimensionless streaming current $\left(I_{d}\right)$ with dimensionless capillary radius $\left(r_{d}\right)$ for the range of values of surface charge and concentration given in Table 2. Each plot corresponds to a different value of concentration; each curve corresponds to a different value of surface charge. In all cases, $I_{d}$ reaches zero at small $r_{d}$ and 1 at large $r_{d}$ (within a tolerance of $0.1 \%$ ), which confirms that the thick double-layer assumption is valid at small $r_{d}(r \ll \lambda)$ and the thin double-layer assumption is valid at large $r_{d}(r \gg$ $\lambda)$. However, at intermediate $r_{d}$, the curves diverge from zero or 1 , demonstrating that there is a range of values of $r$ and $\lambda$ for which neither assumption is valid. At these intermediate values of $r_{d}$, the dimensionless streaming current increases to large values. This reflects the dependence of $\left.I\right|_{\lambda \ll r}$ on $r^{2}$ (12) and $\left.I\right|_{\lambda \gg r}$ on $r^{3}$ (14), which yields a value of $r_{d}$ at which the values of $\left.I\right|_{\lambda \ll r}$ and $\left.I\right|_{\lambda \gg r}$ are identical, and the denominator of (20) falls to zero. An example of this is shown in Figure 4, where the streaming current obtained assuming a thin $\left(\left.I\right|_{\lambda \ll r}\right)$ or thick $\left(\left.I\right|_{\lambda \gg r}\right)$ double layers (using (12) and (24) resp.) is plotted against dimensionless capillary radius $\left(r_{d}\right)$, and compared to that obtained from a full solution of the Poisson-Boltzmann equation. At intermediate values of $r_{d}$, the $\left.I\right|_{\lambda \ll r}$ and $\left.I\right|_{\lambda \gg r}$ deviate from the full solution which shows that the thin and thick double-layer assumptions are not valid. When $\left.I\right|_{\lambda \ll r}$ and $\left.I\right|_{\lambda \gg r}$ intersect, the dimensionless streaming current is undefined because the denominator of 


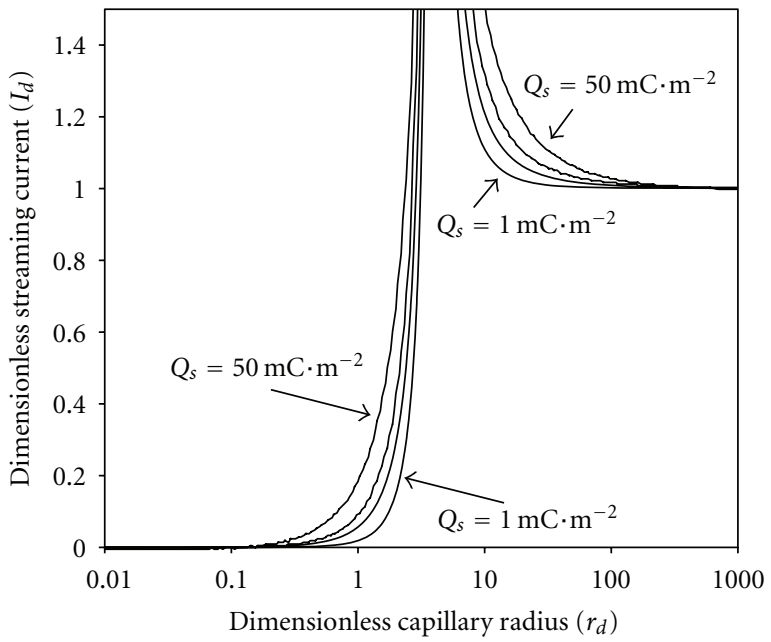

(a)

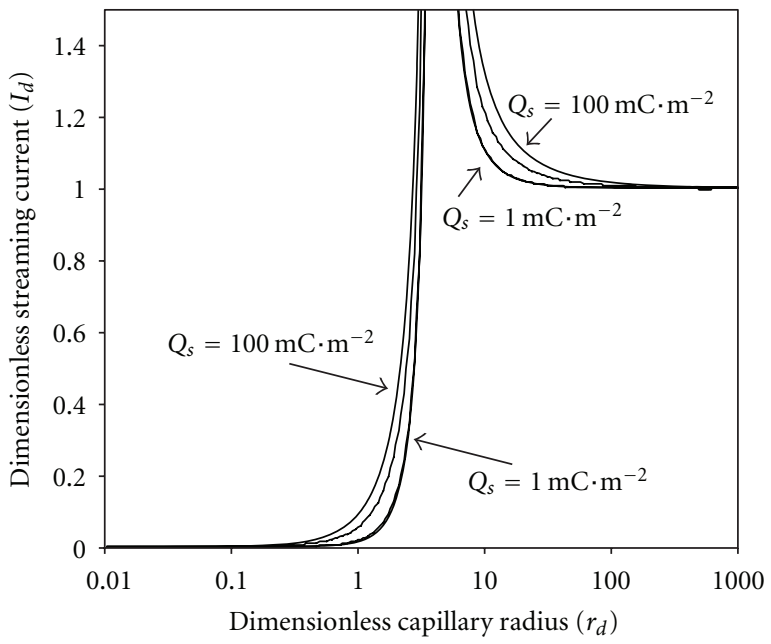

(c)

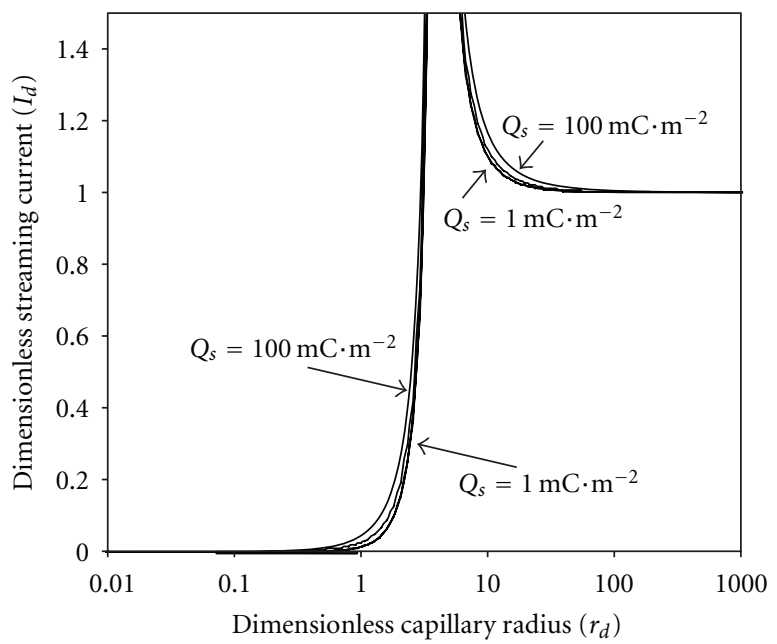

(e)

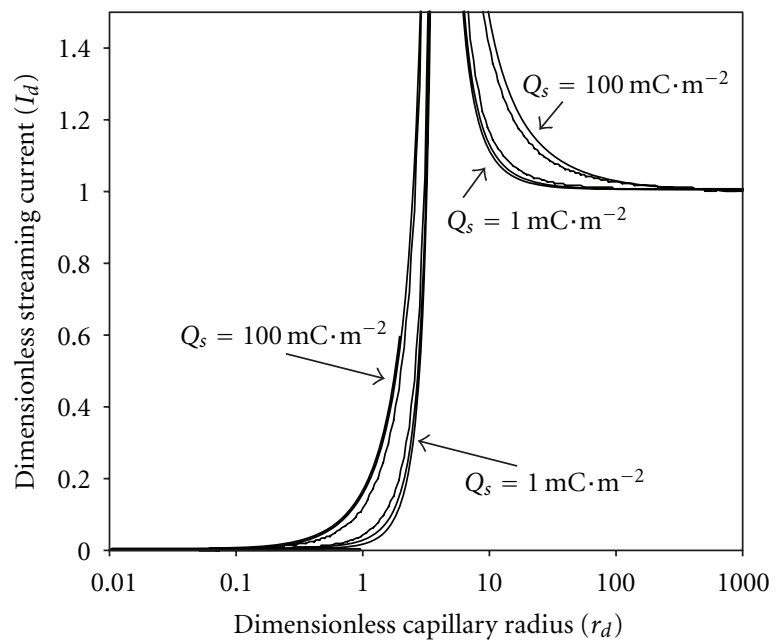

(b)

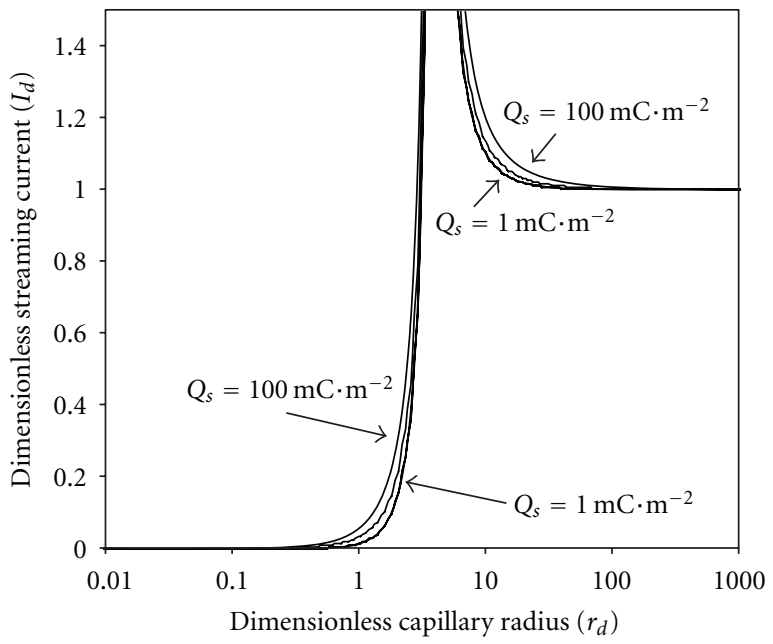

(d)

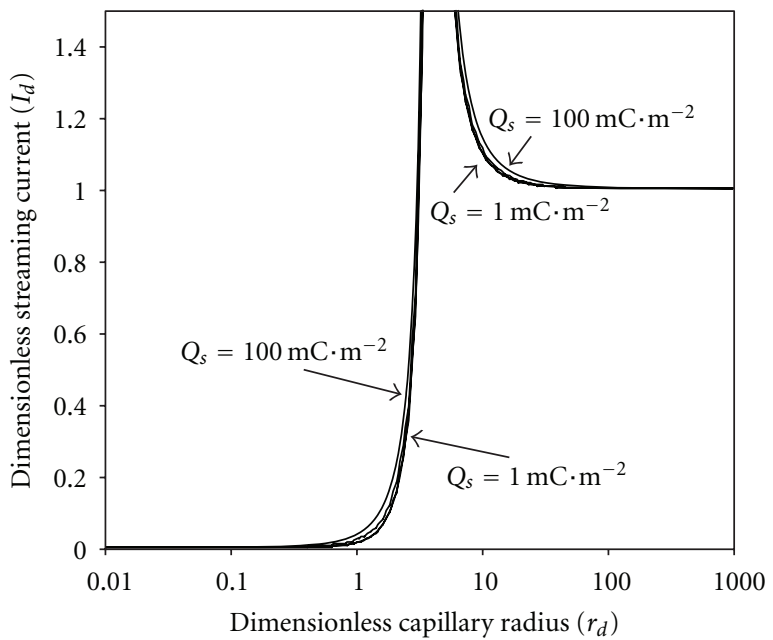

(f)

FIGURE 3: Dimensionless streaming current $\left(I_{d}\right)$ as a function of dimensionless pore radius $\left(r_{d}\right)$ for concentration values of (a) $0.001 \mathrm{M}$; (b) $0.01 \mathrm{M}$; (c) $0.1 \mathrm{M}$; (d) $0.5 \mathrm{M}$; (e) $1 \mathrm{M}$; (f) $2 \mathrm{M}$. Curves denote surface charge values of $1,5,10,50$, and $100 \mathrm{mC} \cdot \mathrm{m}^{-2}$, with curves ordered between the maximum and minimum values indicated on the plots. The $100 \mathrm{mC} \cdot \mathrm{m}^{-2}$ case is not shown in Figure $3(\mathrm{a})$. 
TABLE 2: Values of the properties used in the model.

\begin{tabular}{lcc}
\hline Property & Value & Units \\
\hline$C_{f}$ & $1 \times 10^{-3}-2$ & $\mathrm{M}$ \\
$e$ & $1.60217646 \times 10^{-19}$ & $\mathrm{C}$ \\
$\varepsilon$ & $0.708-0.514$ & $\mathrm{nF} \mathrm{m}^{-1}$ \\
$k$ & $1.38065 \times 10^{-23}$ & $\mathrm{~m}^{2} \mathrm{~kg} \mathrm{~s}^{-2} \mathrm{~K}^{-1}$ \\
$\lambda$ & $9.72-0.185$ & $\mathrm{~nm}^{-1}$ \\
$N_{\mathrm{A}}$ & $6.0221415 \times 10^{23}$ & $\mathrm{Mol}^{-1}$ \\
$Q_{s}$ & $1-100$ & $\mathrm{mC} \mathrm{m}^{-2}$ \\
$T$ & 298.15 & $\mathrm{~K}$ \\
\hline
\end{tabular}

(20) is zero. However, the dimensionless streaming current at intermediate $r_{d}$ has no physical significance, as the calculated values of $\left.I\right|_{\lambda \ll r}$ and $\left.I\right|_{\lambda \gg r}$ are not valid. We are interested here only in determining the range of values of $r_{d}$ for which $I_{d}$ is zero or 1 , to identify the range of validity of the thick and thin double-layer approximations, respectively.

At low concentration (e.g., Figure 3(a)), the range of values of $r_{d}$ for which the thick and thin double-layer assumptions are valid depends on the specific surface charge, with high values of surface charge yielding a smaller range of validity. For example, at the lowest concentration investigated, the thick double-layer assumption is valid (defined as a divergence of $I_{d}$ from 0 of $<1 \%$ ) at low surface charge for $r_{d}<0.81$, while the thin double-layer assumption is valid (defined as a divergence of $I_{d}$ from 1 of $<1 \%$ ) for $r_{d}>24$ (Figure 3(a)). However, at high surface charge, the thick double layer assumption is valid only for $r_{d}<0.17$, while the thin double layer assumption is valid only for $r_{d}>$ 200. As the concentration increases, the effect of varying the specific surface charge decreases. The curves cluster towards the low surface charge case, for which the curves are similar regardless of concentration (Figure 5).

Figure 6 shows the critical value of dimensionless capillary radius for which each assumption is valid (using the definitions given above) over the range of concentration and surface charge investigated. As the electrolyte concentration increases, and the specific surface charge decreases, the critical value of $r_{d}$ below which the thick double layer is validly increases with increasing concentration and decreasing surface charge, from a minimum of 0.17 to a maximum of 0.96. Conversely, the critical value of $r_{d}$ above which the thin double-layer assumption is validly decreases with increasing concentration and decreasing surface charge, from a maximum of 200 to a minimum of 22 .

These results demonstrate that there is not a single value of dimensionless capillary radius below which the thick double layer assumption is valid, or a single value above which the thin double layer assumption is valid; rather the critical dimensionless radius depends upon both concentration and specific surface charge. A conservative estimate of the dimensionless capillary radius below which the thick double-layer assumption is valid, applicable over the range of concentration and surface charge investigated, is $r_{d}<0.17$, while a conservative estimate for the validity of the thin double-layer assumption is $r_{d}>200$. This is equivalent to requiring the capillary radius to be c. 5 times smaller than the diffuse layer thickness for the thick double-layer assumption to be valid, and the capillary radius to be c. 200 larger than the diffuse layer thickness for the thin double layer assumption to be valid. However, at concentrations of $0.1 \mathrm{M}$ and higher, and specific surface charge of $10 \mathrm{mC} \cdot \mathrm{m}^{-2}$ and lower, the thick double layer assumption is typically valid when the pore radius is slightly smaller than the Debye length $\left(r_{d}<0.95\right)$, while the thin double-layer limit is typically valid when the pore radius is c. 25 times larger than the Debye length $\left(r_{d}>25\right)$. We discuss the implications of these results for modelling streaming potentials in natural systems in the next section.

The results presented here can be compared with those of Westermann-Clark and Christoforou [5], who found that the Meyers-Sievers model, in which the excess charge density is assumed to be independent of distance from the pore surface, was a good approximation of the exclusion-diffusion potential across a single capillary regardless of the value of $r_{d}$ (which they termed $a / \lambda$ ) at low surface charge, and when $r_{d}<1$ or $r_{d}>20$ at high surface charge (estimated from Figure 4 of Westermann-Clark and Christoforou [5]). The Meyers-Sievers model is equivalent to the thick double-layer assumption when $r_{d} \rightarrow 0$ but is equivalent to the thin double layer assumption when $r_{d} \rightarrow \infty$ only in the trivial case of a zero streaming current. Our results are similar to those of Westermann-Clark and Christoforou [5] in that we do find critical values of $r_{d}$ for which the thin and thick double-layer assumptions are valid, and these critical values depend on the specific surface charge. However, our results differ from those of Westermann-Clark and Christoforou [5] in a number of ways. Firstly, Westermann-Clark and Christoforou [5] did not investigate or identify any concentration dependence of the critical value of $r_{d}$. Secondly, we always find values of $r_{d}$ for which the thick and thin double-layer assumptions are not valid, regardless of the value of the surface charge investigated (Figure 3). The thin and thick double-layer assumptions only become independent of $r_{d}$ as the surface charge tends to zero, in which case the streaming current tends to zero and the solution is trivial. Finally, we find rather more restrictive limits on the validity of the thin and thick double-layer assumptions at high surface charge (Figure 6). The values of $r_{d}$, for which charge exclusion from the porespace at high surface charge is adequately described by the Meyers-Sievers model, are different from the values of $r_{d}$ for which the streaming current (and, hence, the streaming potential) at high surface charge is adequately described by the thin or thick double-layer assumptions.

We have compared the values of dimensionless streaming current $\left(I_{d}\right)$ that we obtained at large values of $r_{d}$ and $Q_{s}$ using (12), which was derived using the Debye-Hückel model to calculate $Q(x)$, with those obtained using the GouyChapman model (24) to calculate $Q(x)$, and confirmed that the curves are identical within the line widths used in Figure 3. The results are similar because, as $r_{d}$ becomes large, the thickness of the double layer becomes small in comparison to the radius of the capillary, so small differences in $Q(x)$ between the Debye-Hückel and Gouy-Chapman models (e.g., Figure 2(b)) have negligible impact on the calculated 


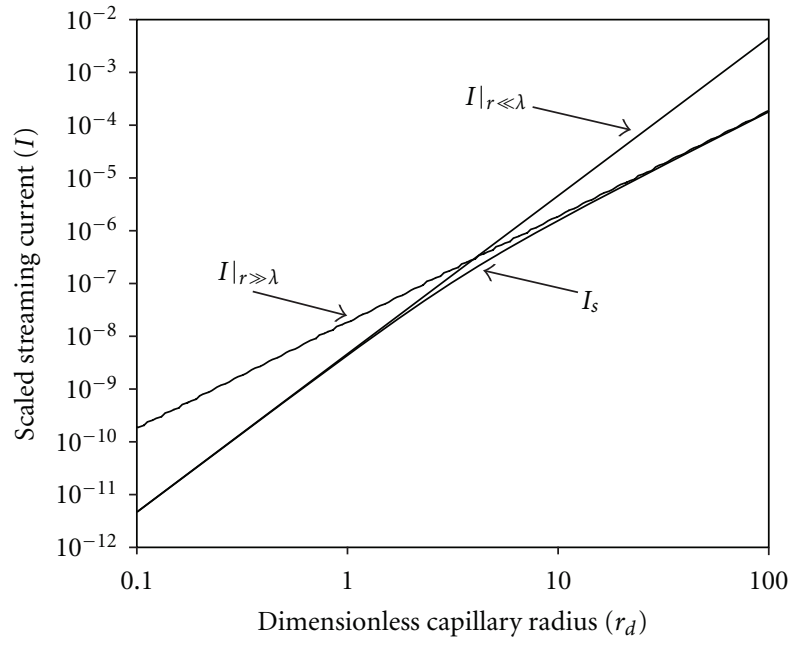

Figure 4: Typical results showing streaming current calculated using (i) the Poisson-Boltzmann equation $\left(I_{s}\right)$, (ii) assuming a thin double layer $\left(\left.I\right|_{\lambda \ll r}\right)$, and (iii) assuming a thick double layer $\left(\left.I\right|_{\lambda \gg r}\right)$, as a function of dimensionless capillary radius $\left(r_{d}\right)$. The reported streaming currents have been scaled to the value of $\left.I\right|_{\lambda \gg r}$ at $r_{d}=$ 1000. Note that there is a value of $r_{d}$ for which $\left.I\right|_{\lambda \gg r}=\left.I\right|_{\lambda \gg r}$. At this value of $r_{d}$, the dimensionless streaming current $I_{d}$ is undefined (20).

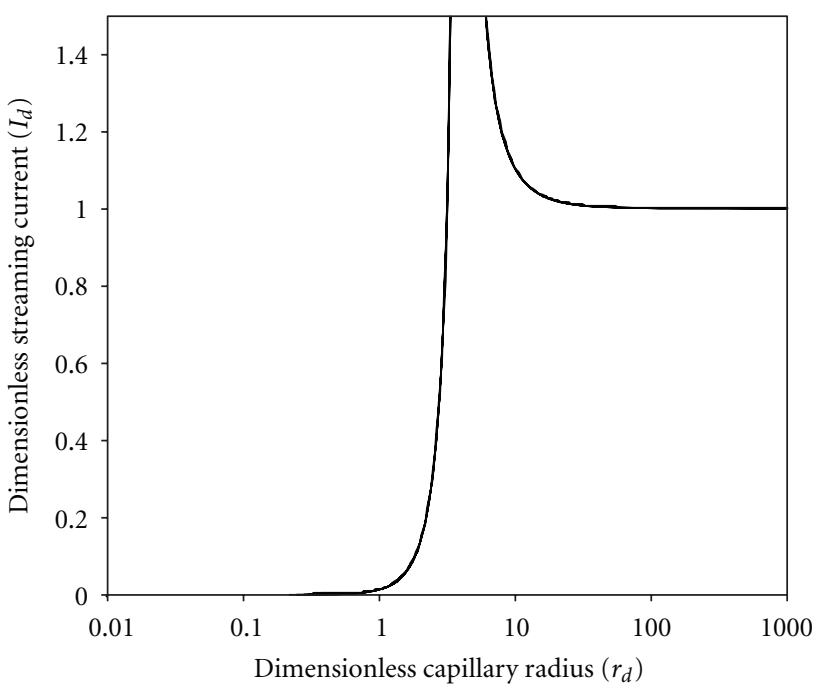

FIGURE 5: Dimensionless streaming current $\left(I_{d}\right)$ as a function of dimensionless capillary radius $\left(r_{d}\right)$ for a surface charge of $Q_{s}$ $=1 \mathrm{mC} \cdot \mathrm{m}^{-2}$. Curves corresponding to concentration values of $0.001 \mathrm{M}, 0.01 \mathrm{M}, 0.1 \mathrm{M}, 0.5 \mathrm{M}, 1 \mathrm{M}$, and $2 \mathrm{M}$ are shown but cannot be distinguished.

streaming current. Even at large $Q_{s},(12)$ provides an appropriate limiting case for the thin double-layer approximation when calculating the dimensionless streaming current.

\section{Discussion}

Our results suggest that in a capillary tubes model, with values of surface charge up to $100 \mathrm{mC} \cdot \mathrm{m}^{-2}$, the thin doublelayer assumption is valid so long as the capillary radius is more than 200 times greater than the thickness of the double layer, while the thick double layer assumption is valid so long as the capillary radius is more than 6 times smaller than the thickness of the double layer. At lower surface charge density $\left(<10 \mathrm{mC} \cdot \mathrm{m}^{-2}\right)$ or higher concentration $(>0.1 \mathrm{M})$, the validity criteria are less stringent: the thin double-layer assumption is valid so long as the capillary radius is more than 25 times the thickness of the double-layer, while the thick double layer assumption is valid so long as the capillary radius is slightly less than the thickness of the double layer. It is interesting to test these criteria for the range of pore size and concentration typical of natural porous media.

Figure 7 shows the (dimensional) critical capillary radius above and below which the thin and thick double-layer assumptions are valid, respectively. The curves were calculated using the data reported in Figure 6 and (2) and (19). Also shown are typical ranges of pore and pore throat sizes in sandstones and mudstones. The pore space topology of capillary tubes model is very different from that of natural sandstones and mudstones (see Jackson [6] for a discussion); however, the results presented here provide firstorder estimates of the validity of the thin and thick doublelayer assumptions in natural porous media. The transport properties of sandstones and mudstones are generally controlled by the connectivity of the larger pores and the size of the connecting throats [7-10], and we assume the same is true of the streaming current, with the capillaries modeled here representing the connecting pore-throats (see also [18]).

The results shown in Figure 7 suggest that at low surface charge $\left(<10 \mathrm{mC} \cdot \mathrm{m}^{-2}\right)$, sandstones lie comfortably in the region for which the thin double-layer assumption is valid, except those with the smallest pore-throat sizes saturated with electrolyte of low concentration. This result supports the assumptions of numerous previous studies of the electrokinetic properties of sandstones (e.g., [13, 14, 23, 32]). However, at higher surface charge, the thin double layer assumption may not be valid in sandstones of moderate- to small-pore-throat size (c. $1-5 \mu \mathrm{m})$, if the electrolyte concentration is less than c. $10^{-3} \mathrm{M}$. It has long been recognized that the classical Helmholtz-Smoluchowski equation (3) fails in sandstones and other porous materials of similar poresize, saturated with electrolytes at low concentration; this is usually attributed to the contribution of surface electrical conductivity, which is neglected in (3) (e.g., $[2,13,14,17$, $33])$. Here we suggest that the thin double-layer assumption, upon which (3) is based, may not be valid in fine-grained or cemented sandstones saturated with low-salinity brine.

The results shown in Figure 7 also suggest that mudstones can lie within the validity range of either the thin or thick double-layer assumption, depending upon their pore-throat size and surface charge, and the electrolyte concentration. However, they may often be poorly described by either assumption. The thin double-layer assumption is likely to be valid at high electrolyte concentration $(>0.2 \mathrm{M})$, regardless of the value of surface charge, if there is wellconnected macroporosity that dominates the transport properties (yielding a majority of pore-throat sizes $>10 \mathrm{~nm}$ ). Moreover, the thick double-layer assumption is likely to be valid in mudstones with pore-throat size greater than a few 


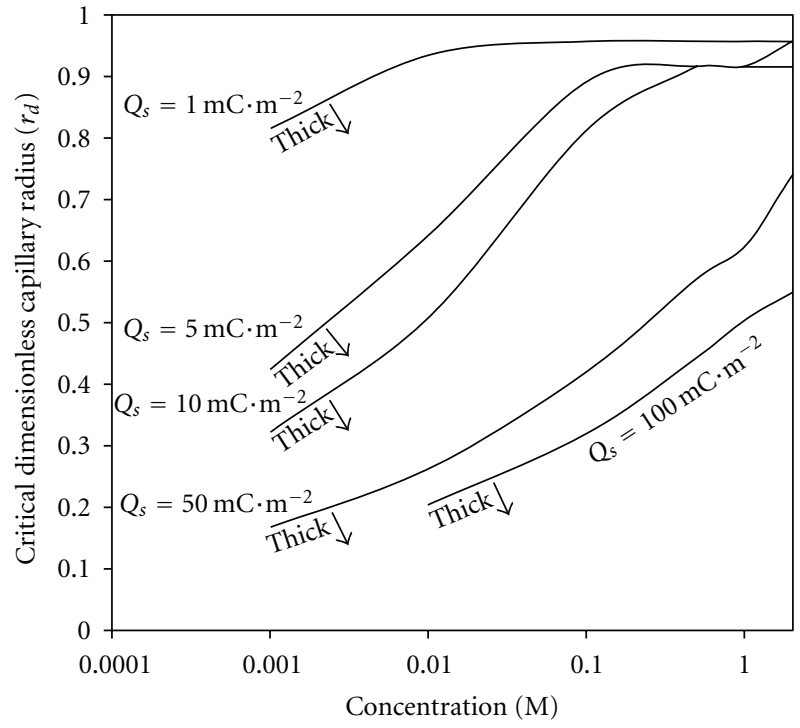

(a)

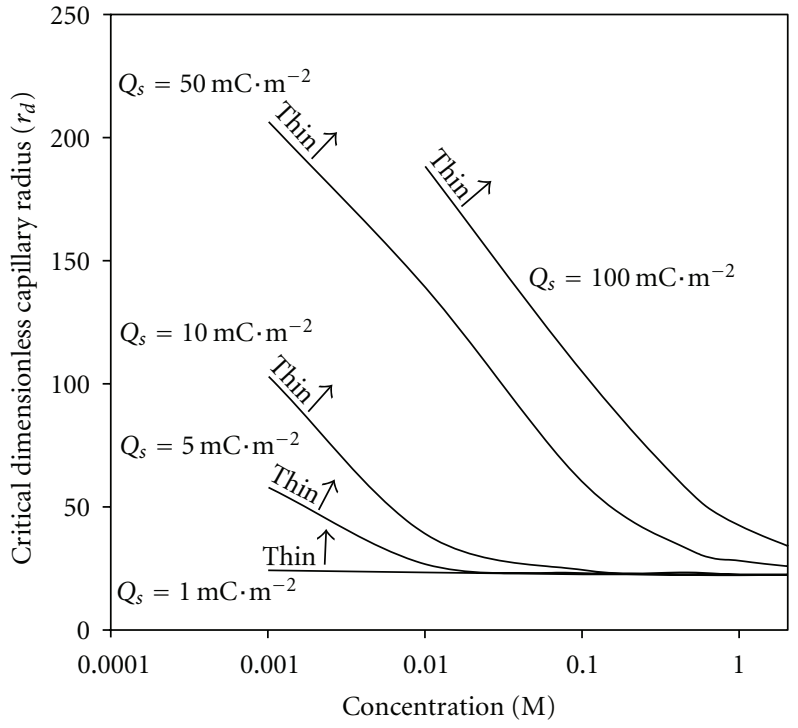

(b)

FIgURE 6: Critical dimensionless capillary radius (determined from Figure 3) (a) below which the thick double-layer assumption is valid and (b) above which the thin double layer assumption is valid, as a function of electrolyte concentration. Curves correspond to surface charge values of $1,5,10,50$, and $100 \mathrm{mC} \cdot \mathrm{m}^{-2}$. The thick double-layer assumption is taken to be valid when the dimensionless streaming current $I_{d}$ deviates from 0 by less than $1 \%$; the thin double-layer assumption is taken to be valid when $I_{d}$ deviates from 1 by less than $1 \%$.

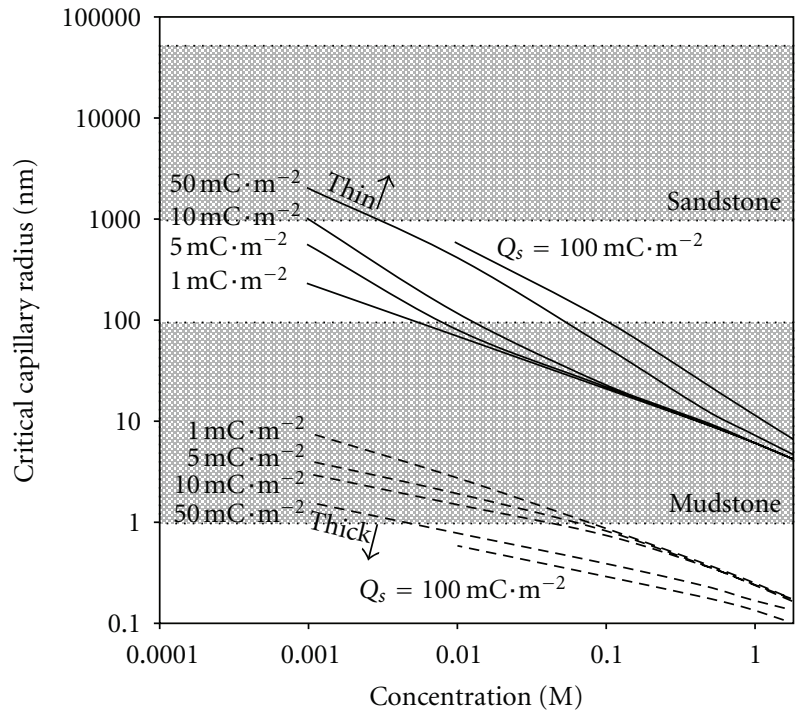

Figure 7: Critical capillary radius (determined from Figure 6) above which the thin double-layer approximation is valid (solid lines) and below which the thick double-layer assumption is valid (dashed lines), as a function of electrolyte concentration. Curves correspond to surface charge values of $1,5,10,50$, and $100 \mathrm{mC} \cdot \mathrm{m}^{-2}$. Also shown are typical ranges of pore and pore-throat radii in sandstones and mudstones [7-10].

nanometres, at electrolyte concentrations below c. $0.1 \mathrm{M}$ and surface charge below $10 \mathrm{mC} \cdot \mathrm{m}^{2}$. This finding supports the approach of Revil and coworkers, who invoke the thick double-layer assumption in their streaming potential model for mudstones and claystones $[18,19,26]$. However, at higher surface charge $\left(>50 \mathrm{mC} \cdot \mathrm{m}^{2}\right)$, the thick doublelayer assumption is likely to be valid in mudstones only at electrolyte concentrations below c. $0.003 \mathrm{M}$, even if the porethroat size is as small as $1 \mathrm{~nm}$. This is a rather restrictive limit in natural systems. Models based on the thick double-layer assumption are never likely to be applicable to sandstones, or other rock types with larger pore and pore-throat sizes than mudstones.

We have derived the validity criteria presented in Figure 7 for a circular capillary tube of uniform radius. As discussed previously, a capillary tube model is a poor approximation for the pore-space topology of real rocks. Here, we assume that charge transport and, hence, the streaming current, is dominated by charge exclusion in the smaller porethroats, rather than the larger pore-bodies, and equate the radius of the capillary tube with the pore-throat radius in real rocks. Extending the analysis to more realistic pore geometries and pore size distributions is outside the scope of this paper. However, we note that Westermann-Clark and Christoforou [5] investigated both circular and slit-like capillary geometries (the slit-like capillary was modeled as two parallel, infinite plates) and found that their criteria for the validity of the Meyers-Sievers model for exclusiondiffusion potentials, in which the excess charge density is assumed to be independent of distance from the pore surface, were the same regardless of the capillary geometry. We suggest that the same is likely to be the case for streaming current calculations. Clearly, there is a need to model more realistic pore-space topologies, but the results we present here provide at least first-order estimates of the validity of the thin and thick double-layer assumptions in natural porous media. 
It should also be noted that we have applied the Poisson-Boltzmann equation (1) to describe the charge distribution in the diffuse layer at electrolyte concentrations up to $2 \mathrm{M}$. It is well known that the assumptions upon which (1) is based begin to break down at high electrolyte concentration (e.g., [2]). Consequently, the criteria we deduce for the thin double-layer limit may be in error. Application of more sophisticated models for the excess charge density, which account for high concentration effects, suggest that corrections to the Poisson-Boltzmann equation are of order $2 \%$ at $0.1 \mathrm{M}$, but may be as large as $25 \%$ at concentrations above $1 \mathrm{M} \mathrm{[2].} \mathrm{However,} \mathrm{we} \mathrm{argue} \mathrm{that}$ high concentration effects impact only on our assessment of the thin double-layer assumption in fine-grained rocks such as mudstones, because the limit of the thin doublelayer assumption in sandstones is only approached at low concentration.

\section{Conclusions}

We find that, for values of surface charge up to $100 \mathrm{mC} \cdot \mathrm{m}^{-2}$, the thin double-layer assumption is valid in a capillary tubes model saturated with a symmetric, monovalent electrolyte, so long as the pore radius is more than 200 times the thickness of the double-layer. The thick double-layer assumption is valid so long as the pore radius is more than 6 times smaller than the thickness of the double layer. At lower surface charge density $\left(<10 \mathrm{mC} \cdot \mathrm{m}^{-2}\right)$ or higher electrolyte concentration $(>0.5 \mathrm{M})$, the validity criteria are less stringent: the thin double-layer assumption is valid so long as the pore radius is more than 25 times the thickness of the double-layer, while the thick double-layer assumption is valid so long as the pore radius is slightly less than the thickness of the double layer.

Applying these criteria to sandstones suggests that if the specific surface charge is less than $10 \mathrm{mC} \cdot \mathrm{m}^{-2}$, the thin double-layer assumption is valid over the range of electrolyte concentrations likely to be encountered in nature and in laboratory experiments. However, at higher surface charge, the thin double-layer assumption may not be valid in sandstones of moderate to small pore-throat size if the electrolyte concentration is less than c. $0.001 \mathrm{M}$. Previous studies have assumed that the Helmholtz-Smoluchowski equation, which follows from the thin double-layer assumption, fails at low electrolyte concentration because of the contribution of surface electrical conductivity. Here, we suggest that the assumption of a thin double layer may be at fault. The thick double-layer assumption is unlikely to be valid in sandstones.

Applying the criteria to mudstones suggests that the thin double layer assumption is likely to be valid if the pore-throat size is greater than c. $10 \mathrm{~nm}$ for electrolyte concentration $>0.1 \mathrm{M}$. The thick double layer assumption is likely to be valid in mudstones at concentrations below c. $0.1 \mathrm{M}$ and surface charge below $10 \mathrm{mC} \cdot \mathrm{m}^{3}$ but, at higher surface charge, it is likely to be valid only at electrolyte concentrations below c. $0.003 \mathrm{M}$. Mudstones often lie in the range where neither the thin nor thick double layer assumption is valid.

\section{Acknowledgments}

The comments of two anonymous reviewers and the associate editor greatly improved the paper.

\section{References}

[1] L. Jouniaux and T. Ishido, "Electrokinetics in Earth sciences: a tutorial," International Journal of Geophysics, vol. 2012, Article ID 286107, 16 pages, 2012.

[2] R. J. Hunter, Zeta Potential in Colloid Science, Academic Press, New York, NY, USA, 1981.

[3] A. Revil, P. A. Pezard, and P. W. J. Glover, "Streaming potential in porous media 1. Theory of the zeta potential," Journal of Geophysical Research B, vol. 104, no. 9, pp. 20021-20031, 1999.

[4] A. Revil, H. Schwaeger, L. M. Cathless III, and P. D. Manhardt, "Streaming potential in porous media 2. Theory and application to geothermal systems," Journal of Geophysical Research B, vol. 104, no. 9, pp. 20033-20048, 1999.

[5] G. B. Westermann-Clark and C. C. Christoforou, "The exclusion-diffusion potential in charged porous membranes," Journal of Electroanalytical Chemistry, vol. 198, no. 2, pp. 213-231, 1986.

[6] M. D. Jackson, "Multiphase electrokinetic coupling: insights into the impact of fluid and charge distribution at the porescale from a bundle of capillary tubes model," Journal of Geophysical Research, vol. 115, Article ID B07206, 2010.

[7] T. Bourbie and B. Zinszner, "Hydraulic and acoustic properties as a function of porosity in Fontainebleau sandstone," Journal of Geophysical Research, vol. 90, no. B13, pp. 11524-11532, 1985.

[8] P. M. Doyen, "Permeability, conductivity, and pore geometry of sandstone," Journal of Geophysical Research, vol. 93, no. 7, pp. 7729-7740, 1988.

[9] F. A. L. Dullien, Porous media: fluid transport and pore structure, Academic Press, San Diego, Calif, USA, 2nd edition, 1992.

[10] A. Hildenbrand, S. Schlomer, and B. M. Krooss, "Gas breakthrough experiments on fine-grained sedimentary rocks," Geofluids, vol. 2, no. 1, pp. 3-23, 2002.

[11] T. Ishido and H. Mizutani, "Experimental and theoretical basis of electrokinetic phenomena in rock-water systems and its applications to geophysics," Journal of Geophysical Research, vol. 86, no. 3, pp. 1763-1775, 1981.

[12] F. D. Morgan, E. R. Williams, and T. R. Madden, "Streaming potential properties of westerly granite with applications," Journal of Geophysical Research, vol. 94, no. 9, pp. 12,44912,461, 1989.

[13] L. Jouniaux and J. P. Pozzi, "Streaming potential and permeability of saturated sandstones under triaxial stress: consequences for electrotelluric anomalies prior to earthquakes," Journal of Geophysical Research, vol. 100, no. 6, pp. 10,19710,209, 1995.

[14] B. Lorne, F. Perrier, and J. P. Avouac, "Streaming potential measurements: 2. Relationship between electrical and hydraulic flow patterns from rock samples during deformation," Journal of Geophysical Research B, vol. 104, no. 8, pp. 1787917896, 1999.

[15] A. V. Delgado, F. Gonzalez-Caballero, R. J. Hunter, L. K. Koopal, and J. Lyklema, "Measurement and interpretation of electrokinetic phenomena," Journal of Colloid and Interface Science, vol. 309, no. 2, pp. 194-224, 2007. 
[16] L. Jouniaux, M. L. Bernard, M. Zamora, and J. P. Pozzi, "Streaming potential in volcanic rocks from Mount Pelee," Journal of Geophysical Research B, vol. 105, no. 4, pp. 8391$8401,2000$.

[17] P. W. J. Glover and N. Déry, "Streaming potential coupling coefficient of quartz glass bead packs: dependence on grain diameter, pore size, and pore throat radius," Geophysics, vol. 75, no. 6, pp. F225-F241, 2010.

[18] A. Revil and P. Leroy, "Constitutive equations for ionic transport in porous shales," Journal of Geophysical Research B, vol. 109, Article ID B03208, 19 pages, 2004.

[19] A. Revil and N. Linde, "Chemico-electromechanical coupling in microporous media," Journal of Colloid and Interface Science, vol. 302, no. 2, pp. 682-694, 2006.

[20] R. E. Rice, "Exact solution to the linearized PoissonBoltzmann equation in cylindrical coordinates," The Journal of Chemical Physics, vol. 82, no. 9, pp. 4337-4340, 1985.

[21] J. F. Dufrêche, V. Marry, O. Bernard, and P. Turq, "Models for electrokinetic phenomena in montmorillonite," Colloids and Surfaces A, vol. 195, no. 1-3, pp. 171-180, 2001.

[22] A. Dukhin, S. Dukhin, and P. Goetz, "Electrokinetics at high ionic strength and hypothesis of the double layer with zero surface charge," Langmuir, vol. 21, no. 22, pp. 9990-9997, 2005.

[23] J. Vinogradov, M. Z. Jaafar, and M. D. Jackson, "Measurement of streaming potential coupling coefficient in sandstones saturated with natural and artificial brines at high salinity," Journal of Geophysical Research B, vol. 115, no. 12, Article ID B12204, 2010.

[24] H. Vink, "Electrokinetics of polyelectrolyte solutions in capillary tubes," Journal of the Chemical Society, Faraday Transactions 1, vol. 84, no. 1, pp. 133-149, 1988.

[25] M. D. Jackson, "Characterization of multiphase electrokinetic coupling using a bundle of capillary tubes model," Journal of Geophysical Research B, vol. 113, no. 4, Article ID B04201, 2008.

[26] A. Revil, W. F. Woodruff, and N. Lu, "Constitutive equations for coupled flows in clay materials," Water Resources Research, vol. 47, Article ID W05548, 21 pages, 2011.

[27] Y. Bernabé, "Streaming potential in heterogeneous networks," Journal of Geophysical Research B, vol. 103, no. 9, pp. 2082720841, 1998.

[28] J. Kierzenka and L. F. Shampine, "A BVP solver based on residual control and the Maltab PSE," ACM Transactions on Mathematical Software, vol. 27, no. 3, pp. 299-316, 2001.

[29] P. M. Dove and C. M. Craven, "Surface charge density on silica in alkali and alkaline earth chloride electrolyte solutions," Geochimica et Cosmochimica Acta, vol. 69, no. 21, pp. 49634970, 2005.

[30] M. Kosmulski and P. Dahlsten, "High ionic strength electrokinetics of clay minerals," Colloids and Surfaces A, vol. 291, no. 1-3, pp. 212-218, 2006.

[31] C. G. Malmberg and A. A. Maryott, "Dielectric constant of water from 0 to $100^{\circ} \mathrm{C}$," Journal of Research of the National Bureau of Standards, vol. 56, no. 1, pp. 1-8, 1956.

[32] M. Z. Jaafar, J. Vinogradov, and M. D. Jackson, "Measurement of streaming potential coupling coefficient in sandstones saturated with high salinity $\mathrm{NaCl}$ brine," Geophysical Research Letters, vol. 36, no. 21, Article ID L21306, 2009.

[33] A. Bolève, A. Crespy, A. Revil, F. Janod, and J. L. Mattiuzzo, "Streaming potentials of granular media: influence of the dukhin and reynolds numbers," Journal of Geophysical Research B, vol. 112, no. 8, Article ID B08204, 2007. 

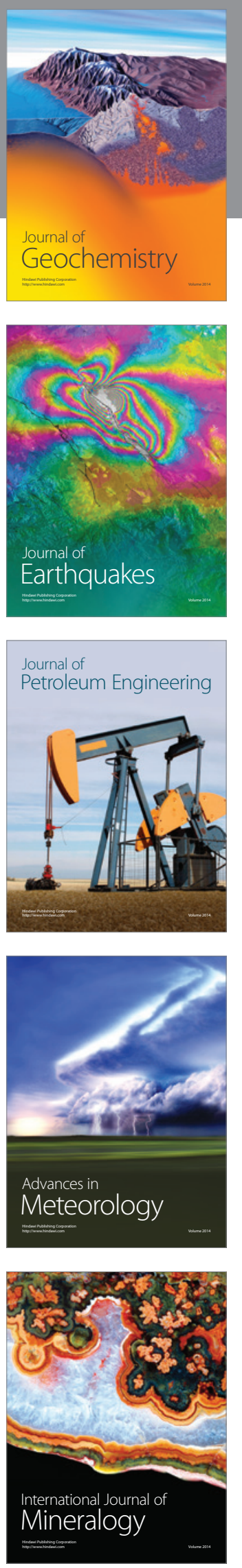
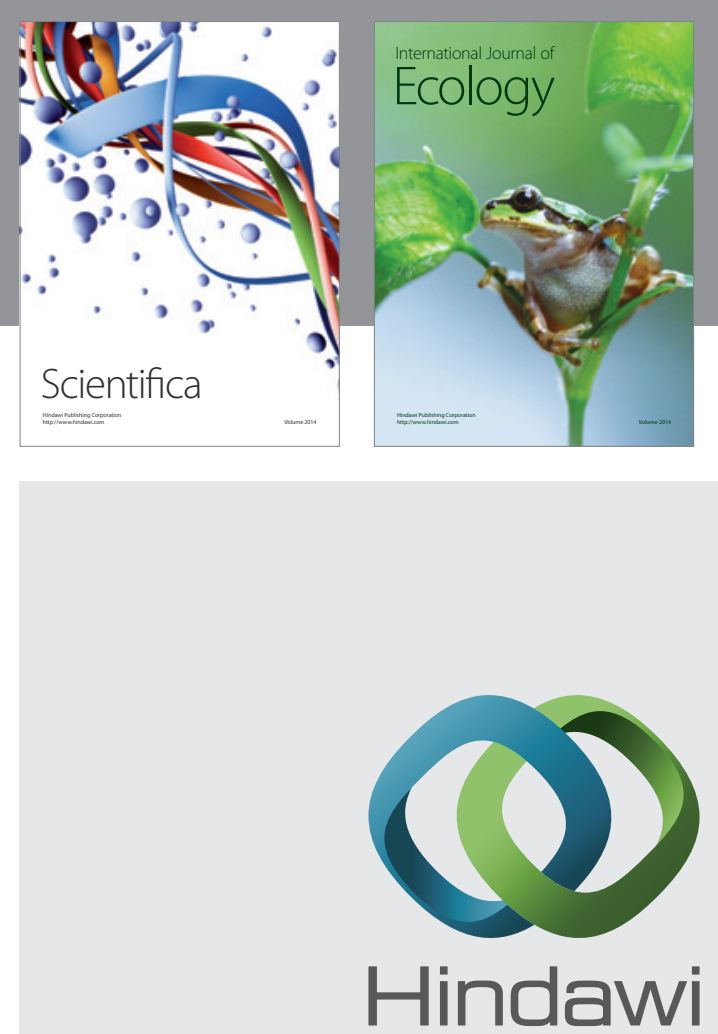

Submit your manuscripts at http://www.hindawi.com
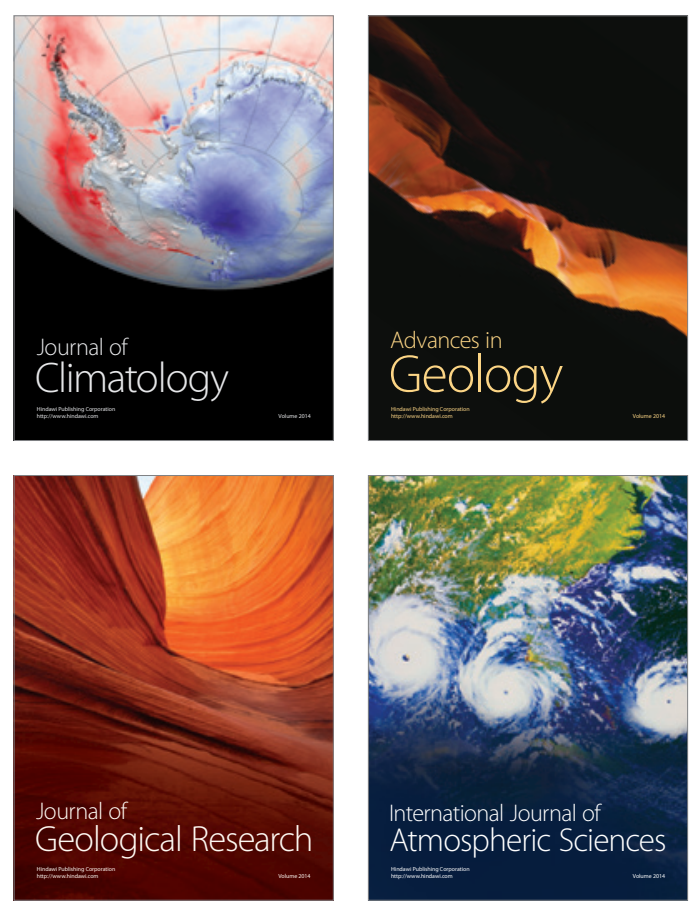
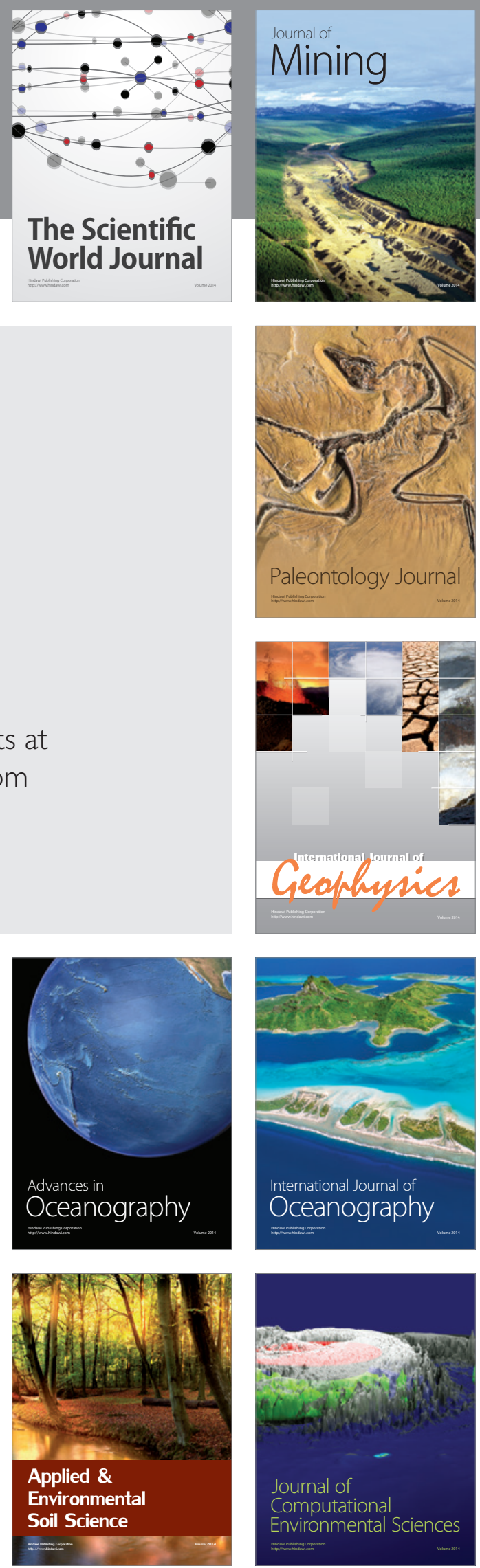